\title{
Enhanced Nodulation and Nodule Development by nolR Mutants of Sinorhizobium medicae on Specific Medicago Host Genotypes
}

\author{
Masayuki Sugawara ${ }^{1}$ and Michael J. Sadowsky, ${ }^{1,2}$ \\ ${ }^{1}$ BioTechnology Institute and ${ }^{2}$ Department of Soil, Water, and Climate, University of Minnesota, St. Paul 55108, U.S.A. \\ Submitted 16 October 2013. Accepted 17 November 2013.
}

\begin{abstract}
The nolR gene encodes a negatively acting, transcriptional regulatory protein of core Nod-factor biosynthetic genes in the sinorhizobia. Although previous reports showed that nolR modulates Nod-factor production and enhances nodulation speed of Sinorhizobium meliloti on alfalfa, there have been no reports for the symbiotic function of this gene in the $S$. medicae-Medicago truncatula symbiosis. Here, we constructed an nolR mutant of $S$. medicae WSM419 and evaluated mutant and wild-type strains for their nodulation ability, competitiveness, host specificity, and densitydependent nodulation phenotypes. When the mutant was inoculated at low and medium population densities, it showed enhanced nodule formation during the initial stages of nodulation. Results of quantitative competitive nodulation assays indicated that an nolR mutant had 2.3fold greater competitiveness for nodulation on $M$. truncatula 'A17' than did the wild-type strain. Moreover, the nodulation phenotype of the nolR mutant differed among Medicago genotypes and showed significantly enhanced nodule development on $M$. tricycla. Taken together, these results indicated that mutation of nolR in $S$. medicae positively influenced nodule initiation, competitive nodulation, and nodule development at later nodulation stages. This may allow nolR mutants of $S$. medicae to have a selective advantage under field conditions.
\end{abstract}

The root and stem nodule bacteria, collectively called the rhizobia, fix dinitrogen gas in symbioses with legume plants. The symbiotic interaction is important for agricultural productivity, especially in less developed countries. However, nodulation ability and competitiveness have practical importance in agricultural production, because the inoculation of efficient rhizobia in often unsuccessful, owing in large part to the presence of more competitive populations of ineffective indigenous rhizobia in soils (Streeter 1994; Toro 1996; Triplett and Sadowsky 1992).

Sinorhizobium meliloti forms symbiotic nitrogen-fixing nodules on Medicago truncatula (barrel medic) and M. sativa (al-

Current address for M. Sugawara: Graduate School of Environmental Science, Hokkaido University, North-10, West-5, Kita-ku, Sapporo, Hokkaido 060-0810, Japan.

Corresponding author: M. J. Sadowsky; E-mail: Sadowsky@umn.edu

* The $e$-Xtra logo stands for "electronic extra" and indicates that three supplementary tables and two supplementary figures are published online.

(C) 2014 The American Phytopathological Society falfa). During this interaction, rhizobial nodulation genes (nod, nol, and noe) are expressed, and synthesize and secrete lipochito-oligosaccharide molecules (Nod-factors) that induce the formation of nodules on the host plant root (Long 1989; Long et al. 1985). The expression of the nodulation genes and nitrogen fixation are regulated in a positive and negative manner for optimal nodulation and efficient plant metabolism in the presence of fixed N (Kondorosi et al. 1989; Loh and Stacey 2003). Positive transcriptional regulation is mediated by the products of the nodD and syrM genes (Long 1996). The NodD protein is activated by specific flavonoid molecules secreted by the host root and seed coat, and interact with upstream nod-box regulatory elements to induce common-, host-, and genotype-specific nodulation genes (Loh and Stacey 2003; Sadowsky et al. 1991).

In $S$. meliloti, negative regulation is mediated by a repressor protein encoded by the nolR gene. This gene was first identified in S. meliloti AK631 as a negative transcriptional regulator of core Nod-factor biosynthetic genes (nodABC) (Kondorosi et al. 1989, 1991). The NolR protein binds to the overlapping promoter region of $\operatorname{nod} D_{1}$ and nodA genes at the RNA polymerase binding region (Cren et al. 1995). Mutants of S. meliloti AK631 lacking an nolR gene overproduced and unsuccessfully decorated Nod factors. This, in turn, resulted in less efficient nodule initiation of alfalfa plants (Kondorosi et al. 1989). Moreover, results from proteomic analyses indicated that the NolR protein likely regulates expression of other genes (Chen et al. 2005).

In $S$. fredii HH103, a close relative of $S$. meliloti which nodulates soybean (Sadowsky et al. 1987; Sugawara et al. 2013), NolR influences symbiosis-related signals, such as secretion of signal-responsive proteins and the production of exopolysaccharides (Vinardell et al. 2004). Thus, it appears that the $S$. meliloti NolR is both a global regulatory protein used to fine turn intracellular metabolism and a key player in symbioses with host leguminous plant (Chen et al. 2000, 2005; Vinardell et al. 2004).

Chen and associates (2005) showed that the expression of the $S$. meliloti nolR gene is regulated in a population-densitydependent manner. The nolA gene, a negative transcriptional regulator of nod genes by Bradyrhizobium japonicum, is also expressed in a population-density-dependent manner and enhances the nodule number on soybean genotypes (Jitacksorn and Sadowsky 2008; Loh and Stacey 2003). However, the influence of nolR on population-density-dependent nodulation in the sinorhizobia has not been investigated thus far. Although rhizobia live in nitrogen-fixing nodules at high cell densities, (approximately $10^{10}$ cells/nodule) (Bergersen 1982), it is expected that nolR is also expressed at high levels. Thus, it is possible that nolR influences not only initial nodulation, as has 
been previously reported (Kondorosi et al. 1989, 1991), but also later stages of nodulation that occur after infection, such as the development of nodule primordia and symbiotic $\mathrm{N}_{2}$ fixation.

A functional nolR gene is conserved in most Sinorhizobium spp. and strains (it is not functional in Rm1021) (Sugawara et al. 2013), and this bacterium generally uses NolR as a regulatory protein for expression of large numbers of genes. Our recent comparative genomics studies showed that Sinorhizobium genomes comprised many accessory genes (Sugawara et al. 2013), some of which likely alter host specificity (Crook et al. 2012). In addition, $S$. medicae and $S$. meliloti are taxonomically related, have differences in gene content, and displayed different nodulation patterns on diverse Medicago host plants (Sugawara et al. 2013). Consequently, we hypothesized that nolR-regulated genes may have different influences on symbiotic performance and intracellular metabolism in $S$. medicae and $S$. meliloti strains.

In this study, we report on the functional characterization of the nolR gene in $S$. medicae. We focused our studies on nodulation competitiveness, host specificity, and population-densitydependent nodulation. We also report on the differences in nolR mutant phenotypes among $S$. medicae and S. meliloti strains on diverse host genotypes.

\section{RESULTS}

Expression of nodC in Sinorhizobium nolR mutant strains.

The nolR mutants of $S$. medicae WSM419 and S. meliloti Rm41 were constructed by insertion of a kanamycin/neomycin resistance gene (aph) into nolR (Supplementary Figs. S1 and S2). To investigate the influence of NolR on expression of Nodfactor biosynthesis, $\beta$-galactosidase assays were done using an nodC-lacZ fusion plasmid (pRmM57). The results show that $\beta$-galactosidase activity (Miller units) by nolR mutants of both species were greater than those seen in the wild-type strains, when $5 \mu \mathrm{M}$ luteolin was added as an inducer in $\mathrm{Y}$ minimal medium (Table 1). The activity level of nodC in the $S$. medicae WSM419 nolR mutant (815.2 \pm 13.1 units) was nearly 4.5-fold greater than that seen in the $S$. meliloti nolR mutant (176.3 \pm 4.4 units) (Table 1). Moreover, the ratio of the activity value (nolR mutant/wild-type) in S. medicae was higher (19.5-fold) than that seen in S. meliloti (6.8-fold). This showed that the $n o l R$ gene in $S$. medicae is a negative regulator of nod gene expression and the regulation of nod gene expression in $S$. medicae is likely tightly regulated by the nolR gene product.

\section{Mutation in S. medicae nolR enhances nodulation speed} on M. truncatula 'A17' and M. sativa 'Agate'.

The influence nolR on symbiotic performance of mutant and wild-type strains was investigated using M. truncatula 'A17' and M. sativa 'Agate'. Results shown in Supplementary Table $\mathrm{S} 1$ indicated that there was no difference between plant phenotypes between wild-type and nolR mutant strains, in either sinorhizobial species, after 4 weeks of incubation.

Table 1. Expression of an nodC-lacZ fusion in wild-type and nolR mutant strains of Sinorhizobium

\begin{tabular}{lcc}
\hline & \multicolumn{2}{c}{ Miller units $^{\mathbf{a}}$} \\
\cline { 2 - 3 } Sinorhizobium strain & With luteolin $^{\mathbf{b}}$ & Without luteolin \\
\hline S. medicae WSM419 wild type & $41.9 \pm 0.6$ & $14.8 \pm 0.7$ \\
S. medicae WSM419 $\Delta$ nolR & $815.2 \pm 13.1$ & $26.7 \pm 2.2$ \\
S. meliloti Rm41 wild type & $25.8 \pm 1.9$ & $17.6 \pm 0.6$ \\
S. meliloti Rm41 $\Delta$ nolR & $176.3 \pm 4.4$ & $19.7 \pm 0.9$ \\
\hline a Values are means \pm standard deviations of data for three biological repli- \\
\multicolumn{2}{l}{ cates. } \\
b Luteolin was added at $5 \mu \mathrm{M}$ final concentration.
\end{tabular}

However, because previous reports showed that a mutation in $S$. meliloti AK631 of nolR leads to a decrease in nodulation speed on $M$. sativa, we investigated whether the nolR mutants made in other sinorhizobial strains and species also influenced the timing of nodule formation at initial stages of nodulation. All Sinorhizobium strains were inoculated onto Medicago seedlings at $10^{7}$ cells per plant, and roots were examined daily for the appearance of nodules. The percentage of Medicago plants nodulated by the wild-type, nolR mutant, and the nolR complemented strains at each day after inoculation is shown in Figure 1. Less time was required for the S. medicae WSM419 nolR mutant strain to initiate nodule formation on $M$. truncatula A17 and M. sativa Agate relative to that seen with the wildtype strain (Fig. 1A and B). Nodules appeared 4 days after the $S$. medicae nolR mutant was first inoculated onto both plants. In contrast, nodulation by the wild-type strain did not begin until 5 days after inoculation (DAI). The proportion of nodulated plant by the nolR mutant was significantly greater $(P<$ $0.05)$ than that seen on plants inoculated with the wild-type strain at 4 and 5 DAI. Moreover, the nodulation speed of the nolR mutant reverted to that of the wild type in the complemented strain, $\Delta$ nolR (pMS20), which harbored the pMS20 plasmid (Fig. 1A and B). Although the nolR mutant nodulated 70 to $80 \%$ of Medicago plants at 5 DAI, only 10 to $30 \%$ of the plants were nodulated by the nolR-complemented strains on both Medicago host plants (Fig. 1A and B). In contrast, mutation of the nolR gene in $S$. meliloti Rm41 did not significantly influence nodulation timing on Agate relative to that seen with the wild-type strain or the nolR-complemented strain harboring pMS19 (Fig. 1C). Because S. meliloti Rm41 formed inef-

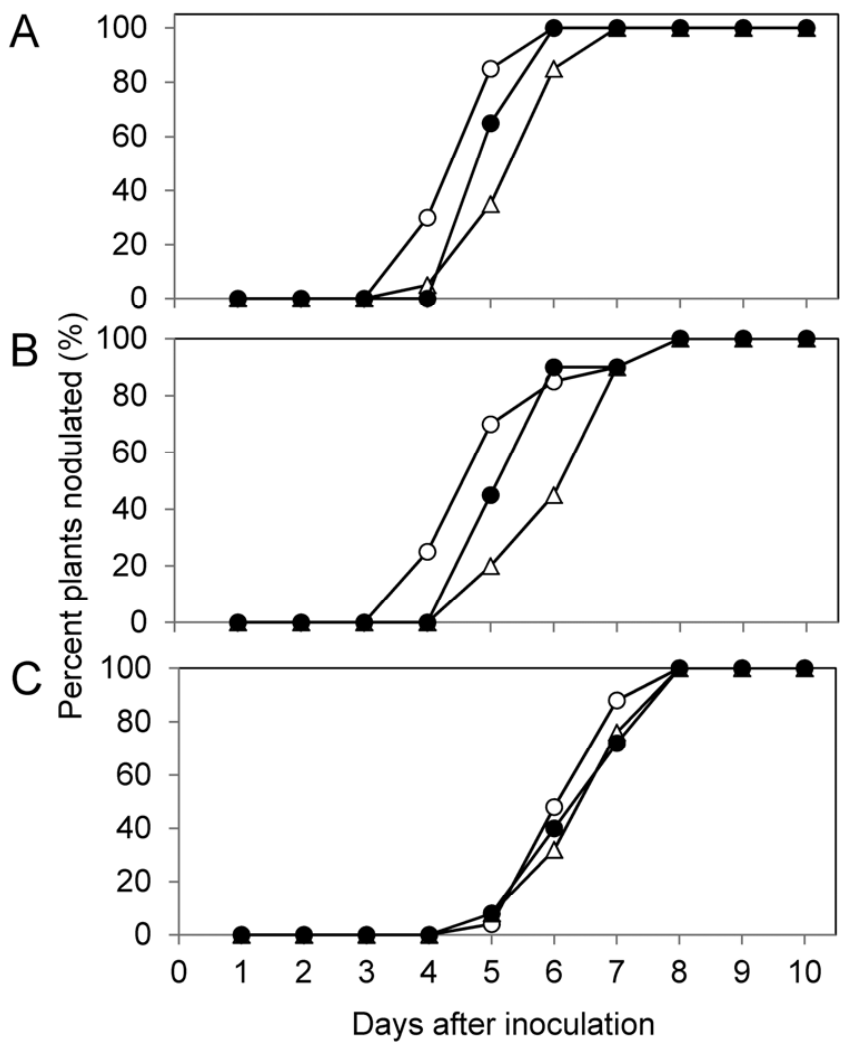

Fig. 1. Percentage of Medicago plants nodulated by a wild-type Sinorhizobium sp. ( $(\bigcirc)$, an nolR mutant $(\bigcirc)$, and its nolR complemented strain $(\triangle)$. A, Percentage of Medicago truncatula 'A17' plants nodulated by Sinorhizobium medicae WSM419 strains; B, percentage of $M$. sativa 'Agate' nodulated by $S$. medicae WSM419; and C, percentage of $M$. sativa Agate nodulated by $S$. meliloti Rm41 strains. Approximately 20 to 25 plants were analyzed for each treatment at each time point. 
fective (small and white-colored) nodules on $M$. truncatula A17, we did not try this experiment using this strain-host combination. Taken together, these results indicated that mutation of the nolR gene in $S$. medicae WSM419 enhanced nodulation at the initial stages of nodulation. Moreover, it suggested that the influence of nolR gene product on the speed of nodulation in S. medicae is greater than that seen in S. meliloti.

\section{The mutation of nolR in S. medicae enhances} competitiveness of nodulation.

The influence of the nolR gene on competitive nodulation ability on Medicago plants was investigated by co-inoculation with the wild-type strain and the nolR mutant. Nodule occupancy rates by each strain are presented in Table 2 . The nodule occupancy rates of the $S$. medicae WSM419 wild-type strain on $M$. truncatula A17 and M. sativa Agate were 27.8 and $23.9 \%$, respectively, when $S$. medicae WSM419 and its nolR mutant were co-inoculated at a 1:1 ratio. In contrast, the nolR mutant showed $72.2 \%$ (A17) and $69.6 \%$ (Agate) nodule occupancy rates. The occupancy rates by the nolR mutant and the wild-type strain were significantly different at $P<0.01$ (Table 2 ). These results indicated that the mutation of nolR in $S$. medicae leads to enhance competitiveness for nodulation. In contrast, no significant influence of nolR gene on $S$. meliloti Rm41 nodule occupancy rates was observed (Table 2).

The competitive ability for nodulation by the nolR mutant of S. medicae on M. truncatula A17 was quantified by using seven inoculant mixtures, with the proportion of wild-type and nolR mutant strains ranging from 1:1,000 to 1,000:1. Nodule occupancy rates by the two strains resulting from each inoculant mixture were obtained and plotted in Figure 2A. In this experiment, the nolR mutant also showed significantly greater nodule occupancy rates $(P<0.01)$ against the wild-type strain when these strains co-inoculated at a $1: 1$ ratio. The relation- ships between the logarithm of the ratio of the numbers of nodules formed by the nolR mutant and the wild-type strain $\left(N_{\Delta n o l R} / N_{\mathrm{WT}}\right)$ and the logarithm of the ratio of the numbers of cells of the two strains in the inoculum $\left(I_{\Delta n o l R} / I_{\mathrm{WT}}\right)$ are shown in Figure 2B. The $C_{\Delta n o l R: W T}$ value for the nolR mutant/wildtype was estimated at 2.29. Thus, this result indicated that the mutation of nolR in S. medicae WSM419 enhances nodulation competitiveness approximately 2.3 -fold on M. truncatula A17.

\section{Mutation of nolR in $S$. medicae enhances nodule development on specific Medicago genotypes.}

The influence of the $S$. medicae nolR gene on host-specific nodulation was investigated by inoculation of wild-type and mutant strains onto nine genotypes of $M$. truncatula and one genotype each of $M$. sativa, $M$. tricycle, and M. littoralis. Nodule numbers and dry weights on each Medicago genotype inoculated with wild-type WSM419 and its nolR mutant are shown in Table 3. These results show that the nolR mutant formed significantly $(P<0.05)$ fewer nodules on the four $M$. truncatula genotypes (SA22322, SA28064, F83005-5, and Salses71B), relative to those seen with the wild-type strain. However, interestingly, the nodule dry weights per plant were not significantly different between the nolR mutant and the wild-type strain, except for F83005-5 (Table 3). In addition, these effects were more clearly observed in $M$. tricycla R108-C3. These results indicated that the nolR mutant formed relatively larger-sized nodules on the Medicago plants than those seen with the wild-type strain. Therefore, we hypothesized that mutation of the nolR gene in $S$. medicae enhances not only initiation of nodulation but also nodule development at later symbiotic stages.

To test this hypothesis, we investigated differentiation of nodule numbers and dry weights on $M$. tricycla $\mathrm{R} 108-\mathrm{C} 3$ at each time point that plants were inoculated with the WSM419 nolR mutant and wild-type strain. Although nodule

Table 2. Competitive nodulation phenotypes of wild-type and nolR mutants of Sinorhizobium medicae WSM419 and S. meliloti Rm41 on Medicago host plants

\begin{tabular}{|c|c|c|c|c|c|c|}
\hline \multirow[b]{2}{*}{ Sinorhizobium strain } & \multirow[b]{2}{*}{ Host plant } & \multicolumn{3}{|c|}{ Nodule occupancy by strain (\%) } & \multirow[b]{2}{*}{$P$ value $^{\mathrm{a}}$} & \multirow[b]{2}{*}{ Nodules tested ${ }^{\mathrm{b}}$} \\
\hline & & Wild type & nolR Mutant & Both & & \\
\hline \multirow[t]{2}{*}{ S. medicae WSM419 } & Medicago truncatula $\mathrm{A} 17$ & 27.8 & 72.2 & 0 & $<0.01$ & 72 \\
\hline & M. sativa Agate & 23.9 & 69.6 & 6.5 & $<0.01$ & 92 \\
\hline S. meliloti $\mathrm{Rm} 41$ & M. sativa Agate & 42.7 & 53.8 & 3.4 & 0.221 & 117 \\
\hline
\end{tabular}

${ }^{\text {a }} P$ values were obtained by $\chi^{2}$ statistical analysis.

${ }^{\mathrm{b}}$ Number of nodules tested.
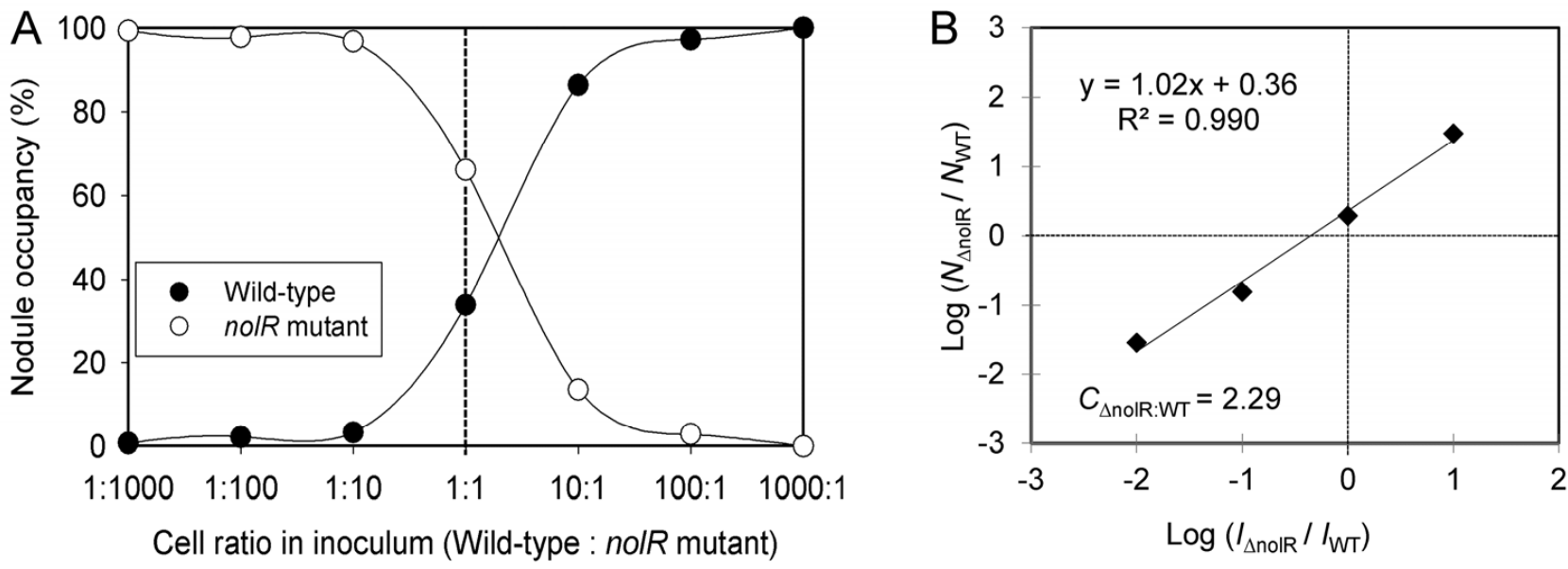

Fig. 2. Quantification of nodule occupancy by co-inoculation of wild-type Sinorhizobium medicae WSM419 and its nolR mutant on Medicago truncatula 'A17'. A, Relationship between percentage of nodule occupancy and cell ratio in inoculum. The $S$. medicae WSM419 wild type (O) and the nolR mutant (O) were co-inoculated on $M$. truncatula A17. The $S$. medicae strains were isolated from each nodule and nodule occupancy was determined by neomycin resistance. Each data point represents the mean of 96 nodules per treatment. B, Relationship between the logarithm of the ratio of the number of nodules formed by the nolR mutant $\left(N_{\Delta n o l R} / N_{\mathrm{WT}}\right)$ and the logarithm of the ratio of the numbers of cells of the two strains in the inoculum $\left(I_{\Delta n o l R} / I_{\mathrm{WT}}\right)$. 
numbers were not significantly different $(P=0.05)$ between the nolR mutant and wild-type strain after 7 and 14 days of inoculation, significantly $(P<0.05)$ fewer nodules were formed on the plants inoculated with the nolR mutant after 21 days (Fig. 3A). In contrast, nodule dry weights were not significantly different $(P=0.05)$ between the strains at each time point (Fig. 3B). However, nodules formed by the nolR mutant on the primary root were relatively larger than that seen with the wild-type strain (Fig. 3D). Moreover, the size and dry weight of the plant inoculated with the nolR mutant were significantly greater $(P<0.05)$ than those seen with the wild-type strain after 42 days (Fig. $3 \mathrm{C}$ and E). These results suggest that mutation of the nolR gene in $S$. medicae enhances nodule development and plant growth on certain Medicago plant genotypes.
The influences of population density on nolR gene expression and nodulation.

To investigate whether the expression of the nolR gene occurs in a population-density-dependent manner, transcriptional nolRgusA gene fusion plasmids were constructed and $\beta$-glucuronidase (GUS) assays were done on cells sampled in the early, middle, and late log phases of growth. GUS activity in both $S$. medicae WSM419(pMS18) and S. meliloti Rm41(pMS17) cells gradually increased with increasing population density, and the greatest activity was observed at the late log phase of growth (Table 4). These results suggested that nolR gene expression in both strains is regulated by a quorum-sensing factor, similar to what was previously reported for the nolR gene in S. meliloti AK631 (Chen et al. 2005) and nolA in B. japonicum USDA 110 (Jitacksorn and Sadowsky 2008; Loh et al. 2002).

Table 3. Nodulation phenotype of wild-type and nolR mutant strains of Sinorhizobium medicae WSM419 inoculated onto several Medicago plant genotypes ${ }^{\mathrm{a}}$

\begin{tabular}{|c|c|c|c|c|c|}
\hline \multirow[b]{2}{*}{ Medicago spp. } & \multirow[b]{2}{*}{ Genotype } & \multicolumn{2}{|c|}{ Nodule number } & \multicolumn{2}{|c|}{ Nodule dry mass (mg) } \\
\hline & & Wild type & nolR mutant & Wild type & nolR mutant \\
\hline \multirow[t]{9}{*}{ M. truncatula } & SA22322 & 22.0 & $12.0^{*}$ & 5.1 & 5.0 \\
\hline & SA28064 & 28.3 & $12.3 *$ & 8.2 & 5.1 \\
\hline & ESP105-L & 12.0 & 13.8 & 3.3 & 2.7 \\
\hline & DZA045-6 & 24.3 & 36.3 & 3.8 & 4.7 \\
\hline & DZA315-16 & 16.3 & 12.0 & 5.0 & 4.2 \\
\hline & F83005-5 & 14.3 & $8.0^{*}$ & 4.4 & $2.7^{*}$ \\
\hline & Salses71B & 13.3 & $8.0 *$ & 2.6 & 2.4 \\
\hline & DZA012-J & 19.8 & 17.0 & 9.9 & 7.0 \\
\hline & A20 & 9.5 & 7.5 & 8.8 & 7.2 \\
\hline M. sativa & Garst6Rm415 & 35.0 & 35.5 & 5.0 & 5.8 \\
\hline M. tricycla & R108-C3 & 10.0 & $2.8^{*}$ & 4.2 & 3.5 \\
\hline M. littoralis & F11013-27 & 0 & 0 & 0 & 0 \\
\hline
\end{tabular}

${ }^{a}$ Values are means of results for four replicate experiments. Nodule number and dry mass values are per plant. Asterisks indicated a significant difference between four biological replicates of the wild-type and nolR mutant strains by analysis of variance at $P<0.05$.
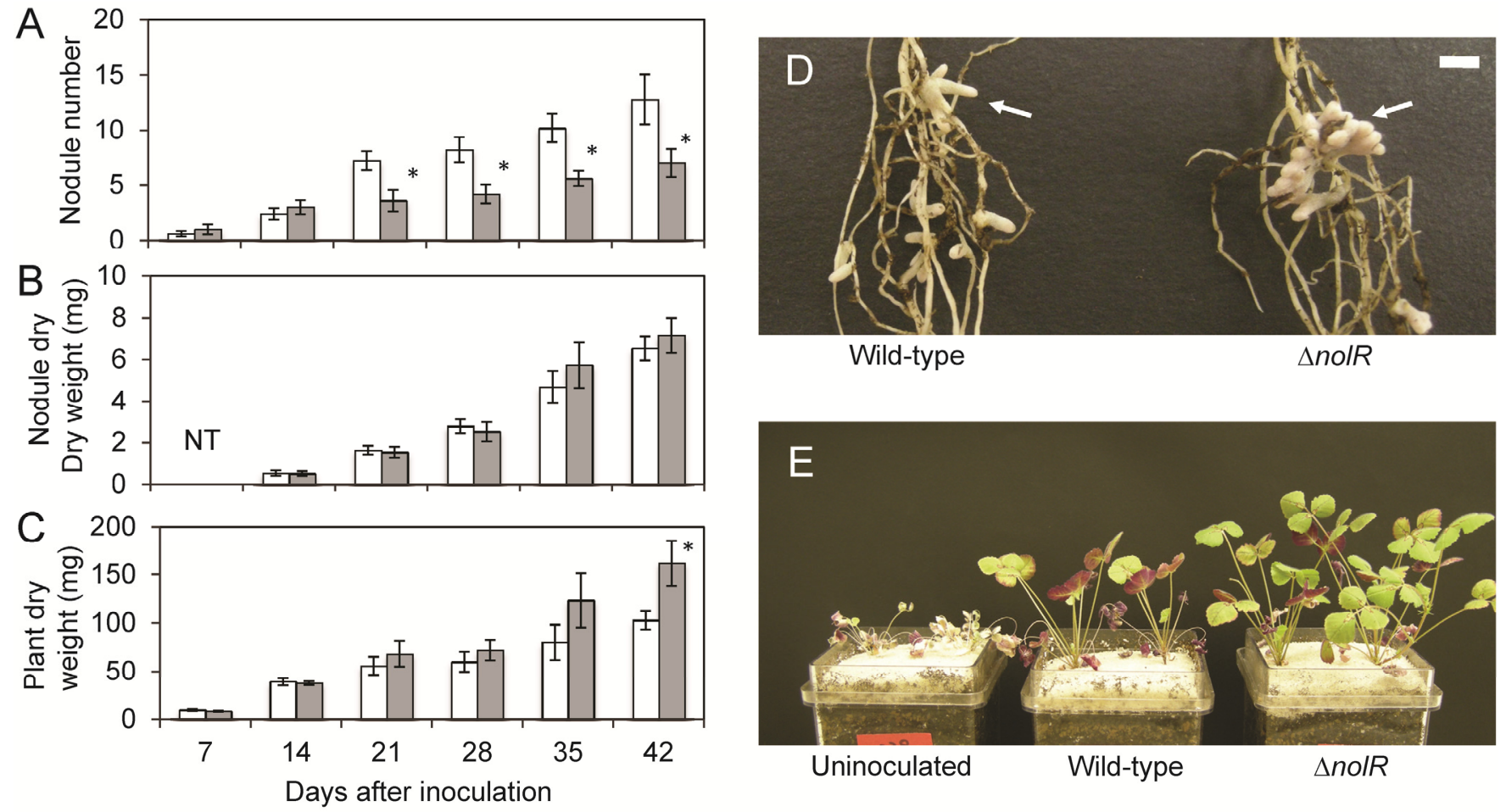

Fig. 3. Symbiotic phenotypes of Medicago tricycla 'R108-C3' inoculated with wild-type and nolR mutant strains of Sinorhizobium medicae WSM419. A, Number of nodules formed; $\mathbf{B}$, nodule dry weight; and $\mathbf{C}$, plant dry weight per plant were plotted at each time point. Asterisks indicate significant difference between the wild type and the nolR mutant at $P=0.05$ as tested by analysis of variance. Error bars indicate standard deviation of data for six replicates. NT $=$ not tested. D, Nodules formed on $M$. tricycla R108-C3 plant roots inoculated with wild-type and nolR mutant strains of S. medicae WSM419. White arrows indicate nodules formed on primary root of $M$. tricycla after 42 days of inoculation. Bar $=0.5 \mathrm{~cm}$. E, $M$. tricycla R108-C3 plants inoculated with the wild type and nolR mutants of $S$. medicae WSM419 and a noninoculated control after 42 days of incubation. 
We investigated population-density-dependent nodulation of M. truncatula A17 by inoculating $S$. medicae WSM419 and its nolR mutant at $10^{3}, 10^{5}, 10^{7}$, or $10^{9}$ cells per plant. First nodules appeared 6 DAI when the wild-type strain was inoculated at $10^{3}$ cells and at 5 DAI when plants received inoculation of $10^{5}$ cells (Fig. 4A). Plants formed nodules earliest, after 4 days, when inoculated with $10^{7}$ or $10^{9}$ cells. Moreover, $100 \%$ of M. truncatula A17 seedlings showed nodulation at 5 DAI, the earliest seen, when inoculated with $10^{9}$ cells. Thus, nodulation by $S$. medicae WSM419 was enhanced with increasing population density. When the nolR mutant was inoculated at $10^{3}$ to $10^{7}$ cells per plant, nodulation speed increased with increasing population density, similar to what was seen with the wild-type strain. However, nodulation was slower when the mutant was inoculated at $10^{9}$ cells compared with the $10^{7}$-cell inoculum (Fig. 4B). Thus, the nodulation speed by the nolR mutant was reduced when the strain was inoculated at high population density.

The root tip marking (RTM) technique (Bhuvaneswari et al. 1980) was used to investigate nodule-forming speed and efficiency at different cell densities. Nodule distances of the wildtype strain gradually increased with increasing population density (Fig. 4C). In contrast, although plants inoculated with the $n o l R$ mutant had nodules that increased in distance from the RTM with increasing cell density from $10^{3}$ to $10^{7}$ cells, distance to the RTM decreased when the nolR strain was inoculated at $10^{9}$ cells. These results are consistent with results of previous nodulation timing assays (Fig. 4A and B). Taken to-

Table 4. Growth phase-dependent expression of an nolR -gusA fusion in Sinorhizobium medicae WSM419 and S. meliloti Rm41

\begin{tabular}{lcclcc}
\hline & \multicolumn{2}{c}{ S. medicae $\mathbf{~ W S M 4 1 9}$} & & \multicolumn{2}{c}{ S. meliloti Rm41 } \\
\cline { 2 - 3 } \cline { 5 - 6 } Growth phase & $\mathbf{O D}_{\mathbf{6 0 0}}{ }^{\mathbf{a}}$ & GUS (units) $^{\mathbf{b}}$ & & $\mathbf{O D}_{\mathbf{6 0 0}}{ }^{\mathbf{a}}$ & GUS (units) \\
\hline Early $\log$ & 0.195 & $378 \pm 9.8$ & & 0.179 & $426 \pm 16$ \\
Middle $\log$ & 0.764 & $530 \pm 3.5$ & & 0.789 & $534 \pm 26$ \\
Late $\log$ & 1.685 & $1,004 \pm 27$ & & 1.480 & $786 \pm 52$ \\
\hline
\end{tabular}

${ }^{\mathrm{a}} \mathrm{OD}_{600}=$ optical density at $600 \mathrm{~nm}$. Strains were grown in tryptone-yeast extract medium.

${ }^{\mathrm{b}} \beta$-Glucuronidase (GUS) activity. Values are means \pm standard deviations of data for four biological replicates. $\beta$-Glucuronidase activity (unit) was expressed as $1,000 \times \mathrm{OD}_{405} / \mathrm{OD}_{600} /$ volume $(\mathrm{ml}) /$ reaction time $(\mathrm{min})$. gether, these results indicate that mutation of the $S$. medicae $n o l R$ gene enhanced nodulation speed at lower population density but inhibited nodulation at high population density.

\section{DISCUSSION}

Although the presence of nolR has been known for years, it is naturally disrupted by a single-base insertion in strain Rm1021, the first sequenced Sinorhizobium strain (Cren et al. 1994). Previous reports showed that the lack of an nolR gene in S. meliloti AK631 leads to less efficient nodule initiation on $M$. sativa $\mathrm{L}$. var. Nagyszénás (Kondorosi et al. 1989). In contrast, results of our studies indicate that a mutation of the $S$. medicae WSM419 $n o l R$ gene significantly enhances initiation and competition for nodulation on both M. truncatula A17 and M. sativa Agate, when the Sinorhizobium cells were inoculated at $10^{7}$ cells per plant (Fig. 1; Table 2). Although the mechanism by which this occurs is presently unknown, the differences in nodulation phenotypes between $S$. medicae WSM419 and S. meliloti AK631 may be due to differences in the plant genotypes tested and bacterial species or inoculant density used. Interestingly, NolR in some rhizobial species acts in a plant genus-specific manner. For example, inactivation or overexpression of nolR in $S$. fredii HH103 decreased the capacity of the strain to nodulate Glycine max but enhanced its ability to nodulate Vigna unguiculata (cowpea) (Vinardell et al. 2004). In addition, our results also show that nodule initiation on M. truncatula A17 was inhibited when nolR mutant cells were inoculated at high population density (Fig. 4). Taken together, the alterations of efficiency of initial nodulation by $n o l R$ are likely dependent on the rhizobial strain and legume used and bacterial population density in soil.

Results from the studies presented here also show that mutation of nolR in S. medicae WSM419 enhances nodule development on the roots of specific Medicago plant genotypes (Table 3; Fig. 3). Thus, these results suggest that the nolR in $S$. medicae WSM419 is involved in host-specific nodulation, and suppresses the expression of unknown symbiosis-related genes at a later stage of nodulation on the genotypes. Although the molecular mechanisms of enhancement of nodule development by the nolR gene remain thus far unresolved, it is a clear example of a new function for the sinorhizobial nolR gene on symbiotic
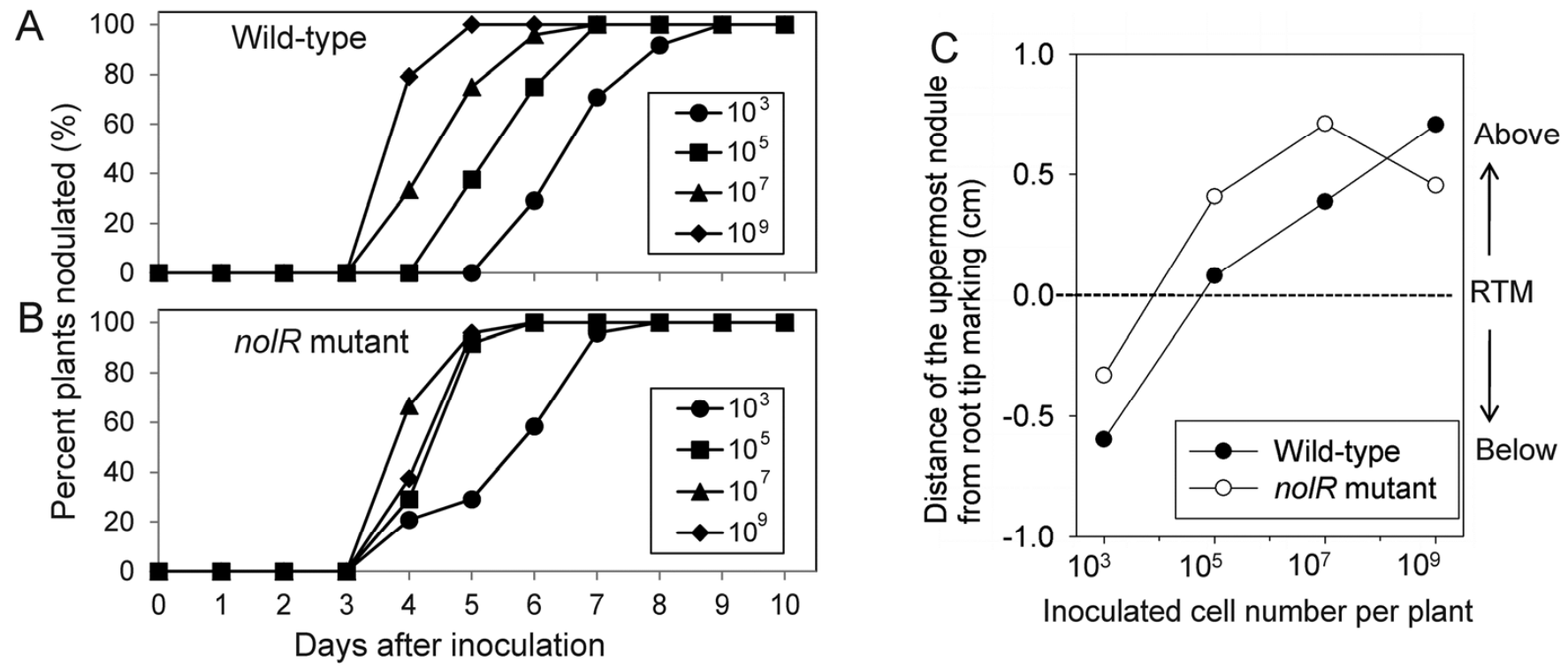

Fig. 4. Percentage of Medicago truncatula 'A17' plants nodulated by wild-type and nolR mutant strains of Sinorhizobium medicae WSM419 at different population densities. The percentage of Medicago plants nodulated by A, wild-type $S$. medicae WSM419 and B, the nolR mutant at each time point is shown. C, Influence of population density on distance of uppermost nodule from the root tip (RT) mark by the $S$. medicae WSM419 wild-type ( ) and the nolR mutant $(\bigcirc)$ strains. Twenty-four plants from each treatment were analyzed at each time point. 
phenotypes at later nodulation stages. Further study of this phenomenon using transcriptomic or proteomic approaches on WSM419 nolR mutant bacteroids may shed further light on new factors involved in nodule development in the MedicagoSinorhizobium symbiosis.

Our results also show that expression of nolR in $S$. medicae WSM419 is enhanced with increasing population density, similar to that reported for $S$. meliloti $\mathrm{Rm} 41$ (Table 4). In addition, a previous study showed that the addition of supernatant of stationary-phase culture of $S$. meliloti AK631 also induced the expression of nolR (Chen et al. 2005). Thus, these results indicate that a similar quorum-sensing system, and possibly signal molecules, may be acting the same in both species and that this is not a strain-specific phenomenon. Several $\mathrm{N}$-acetyl homoserine lactone (AHL)-type quorum signals and the responsible genes have been identified in the rhizobia (Pongslip et al. 2005; SanchezContreras et al. 2007), and a non-AHL-type quorum signal, bradyoxetin, is produced by $B$. japonicum USDA110, which enhances nolA (a negatively acting genotype-specific nodulation gene of soybean) expression at higher population density (Loh et al. 2002). Identification of these nolR-inducing quorum signal molecules from culture supernatants would be useful to better understand symbioses with this host plant.

Nodulation competitiveness has practical importance in agriculture, because the inoculation of efficient rhizobia in often unsuccessful owing to the presence of more competitive but inferior $\mathrm{N}_{2}$-fixing populations in the soil (Streeter 1994; Toro 1996; Triplett and Sadowsky 1992). Several rhizobial factors that affect nodulation competitiveness are known, including motility (Liu et al. 1989), rhizopines (Gordon et al. 1996), exopolysaccharides (McDermoti and Graham 1990), 1-aminocyclopropane-1-carboxylalte deaminase (Uchiumi et al. 2004), and rhizobitoxine (Yuhashi et al. 2000). In addition, of these factors, we found here that mutation of nolR significantly enhanced nodulation efficiency and competitiveness of strains on $M$. truncatula and M. sativa. Moreover, the positive effects of the nolR mutation were observed when the sinorhizobial cells were inoculated at lower population density $\left(10^{3}\right.$ to $10^{7}$ cells per plant). Taken together, the mutation of nolR in $S$. medicae enhances symbiotic performance, and this may help improve the use of this bacterium as a legume inoculant for Medicago plants.

Finally, previous proteome analyses suggested that the nolR gene of $S$. meliloti AK631 encodes a negatively acting transcriptional regulator of core Nod-factor biosynthetic genes, and also acts as a global regulatory protein in response to environmental factors and to fine tune intracellular metabolism (Chen et al. 2000, 2005). In the results presented here, we showed that the nolR gene in $S$. medicae is also a negative regulator of nod gene expression and the regulation of nod gene expression in $S$. medicae is likely more tightly regulated by nolR gene product than that seen in $S$. meliloti. In addition, results from carbon source utilization assays by Biolog GN2 plate (Biolog Inc., Hayward, CA, U.S.A.) suggest that the $S$. medicae NolR also globally regulates intracellular metabolic genes (data not shown). Taken together, these results suggest that NolR may globally control gene expression in $S$. medicae more tightly than in S. meliloti, and $n o l R$-regulated genes likely may be different in $S$. medicae and $S$. meliloti, because these taxonomically related species have large gene content differences (Sugawara et al. 2013).

\section{MATERIALS AND METHODS}

\section{Bacterial strains, plasmids, oligonucleotide primers,} and growth media.

Bacterial strains and plasmids used in this study are shown in Supplementary Table S2. The oligonucleotide primers used in this study are shown in Supplementary Table S3. The Sinorhizo- bium strains were grown at $30^{\circ} \mathrm{C}$ in tryptone-yeast extract (TY) medium (Beringer 1974), and Escherichia coli strains were grown at $37^{\circ} \mathrm{C}$ in Luria-Bertani (LB) medium (Sambrook and Russell 2001). Growth media were supplemented with antibiotics, as required, at the following concentrations (per milliliter) for Sinorhizobium: $150 \mu \mathrm{g}$ of neomycin $(\mathrm{Nm}), 25 \mu \mathrm{g}$ of tetracycline (Tet), and $20 \mu \mathrm{g}$ of gentamicin (Gm); and, for E. coli, $100 \mu \mathrm{g}$ of ampicillin, $50 \mu \mathrm{g}$ of kanamycin, and $25 \mu \mathrm{g}$ of Tet.

\section{Construction of nolR mutants}

\section{of S. medicae WSM419 and S. meliloti Rm41.}

Mobilizable nolR inactivation plasmids were constructed as follows. The 1.2-kb nolR gene and its franking regions from both $S$. medicae WSM419 and $S$. meliloti Rm41 strains were amplified by polymerase chain reaction (PCR) using the oligonucleotide primers WSM419_nolR_PstIF and WSM419_ nolR_PstIR (for S. medicae WSM419) or Rm41_nolR_PstIF and Rm41_nolR_PstIR (for S. meliloti Rm41). The PCR products contained newly created PstI sites. The PCR products were digested by PstI and ligated into pBlueScript SKII (+) to create pMS02 (for the $S$. medicae WSM419 nolR) and pMS01 (for the $S$. meliloti Rm41 nolR). These plasmids were digested by $N c o$ I and the 1.2-kb aph cassette, obtained from pUC4K (Vieira and Messing 1982) by PCR using the oligonucleotide primers (aph_NcoIF and aph_NcoIR), was inserted into nolR to create plasmids pMS08 (WSM419 nolR::aph) and pMS07 (Rm41 nolR::aph). Plasmids pMS08 or pMS07 were digested by $P s t \mathrm{I}$ and the 2.4-kb fragment containing nolR::aph region was inserted into Pst I site in the suicide vector pSUP202 (Simon et al. 1983) to create pMS11 (for Rm41) and pMS12 (for WSM419). The plasmids pMS11 or pMS12 were introduced into $S$. meliloti $\mathrm{Rm} 41$ or $S$. medicae WSM419, respectively, by triparental mating. Mutated strains were selected on TY agar plates containing $\mathrm{Gm}$ at $20 \mu \mathrm{g} / \mathrm{ml}$ and $\mathrm{Nm}$ at $150 \mu \mathrm{g} / \mathrm{ml}$. Gene replacement double-crossover mutants were verified by their antibiotic resistance phenotype $(\mathrm{Gm}$ and $\mathrm{Nm}$ resistant and Tet sensitive), PCR amplification using primers that spanned the insertion sites, and DNA sequence analysis.

\section{Construction of nolR-complemented strains.}

To complement nolR mutants, full-length nolR, including the predicted native nolR promoter and Shine-Dalgarno sequences, was amplified by PCR using Sinorhizobium genomic DNA as template and oligonucleotide primers nolRN_all_PstI and nolRC_all_BglII. The PCR product was digested by PstI and $B g l \mathrm{II}$ and inserted into the PstI-BglII site of pFUS1 (Reeve et al. 1998) to create pMS19 (for Rm41 nolR) and pMS20 (for WSM419 nolR). Plasmids pMS19 or pMS20 were introduced into the S. meliloti Rm41 nolR and S. medicae WSM419 nolR mutants by triparental mating. The nolR-complemented strains were selected on TY agar plate containing Gm, Nm, and Tet.

Evaluation of nodC gene expression by $\beta$-galactosidase assay.

Sinorhizobium wild-type and nolR mutant strains harboring the nodC-lac $Z$ transcriptional fusion plasmid pRmM57 were assayed for $\beta$-galactosidase activity, as previously described (Mulligan and Long 1985). Cultures were washed twice in Y minimal medium (Beringer 1974) and adjusted to an optical density at $600 \mathrm{~nm}\left(\mathrm{OD}_{600}\right)$ of approximately $0.5,5 \mu \mathrm{M}$ luteolin was added, and the cultures was incubated for $4 \mathrm{~h}$ at $30^{\circ} \mathrm{C}$. Units of $\beta$-galactosidase activity were measured using the Miller method (Miller 1972).

\section{GUS assay for evaluation}

of population-density-dependent nolR gene expression.

The nolR gene expression levels in Sinorhizobium strains harboring the nolR-gusA transcriptional fusion plasmids pMS17 
(for S. meliloti Rm41) and pMS18 (for S. medicae WSM419) were assayed for GUS activity, as described by Reeve and associates (1998). Cultures were washed twice in TY medium, adjusted to an $\mathrm{OD}_{600}$ of approximately 0.05 , and $3 \mathrm{ml}$ of the suspension was incubated at $30^{\circ} \mathrm{C}$. Aliquots $(100 \mu \mathrm{l})$ of cells sampled at each phase of growth were mixed with $790 \mu$ l of GUS assay buffer (50 mM sodium phosphate [pH 7], $50 \mathrm{mM}$ dithiothreitol, and $1 \mathrm{mM}$ EDTA) and $100 \mu \mathrm{l}$ of saline. For a blank, 100 $\mu \mathrm{l}$ of saline was added instead of cells. One drop of toluene was added to each tube, the mixture was vortexed, and the tubes were incubated at $37^{\circ} \mathrm{C}$ for $30 \mathrm{~min}$ to remove toluene. The GUS assays were done as previously described (Reeve et al. 1998).

\section{Symbiotic phenotypes of Sinorhizobium wild-type and mutant strains.}

Medicago seed were prepared as described by Bucciarelli and associates (2006). Plant assays were done in sterile Leonard jar assemblies containing a 1:1 mixture of Sunshine mix number 5 (SunGro Horticulture Inc., Vancouver, BC Canada) and Turface MVP (Profile Product LLC, Buffalo Grove, IL, U.S.A.), as previously described (Sugawara et al. 2013). Sinorhizobium strains were grown in TY medium for 2 days, collected by centrifugation at $8,000 \times g$ for $10 \mathrm{~min}$, and washed twice with sterile $0.85 \%$ sodium chloride. The resultant cultures were diluted to $10^{7}$ cells $/ \mathrm{ml}$ with sterile $0.85 \% \mathrm{NaCl}$ and aliquots $(1 \mathrm{ml})$ of serial, decimal dilutions of this inoculum were added onto sterilized Medicago seedlings. Plants were watered with nitrogenfree plant nutrient solution (Bucciarelli et al. 2006) and incubated in a plant growth chamber at $25^{\circ} \mathrm{C}$ with a 16-h light condition and at $21^{\circ} \mathrm{C}$ for $8 \mathrm{~h}$ in the dark. Nodule numbers, dry weights, and dry weight of each plant were determined 1 to 6 weeks after inoculation, as required.

\section{Competitive nodulation assay.}

Sterilized Medicago seedlings were inoculated with $1 \mathrm{ml}$ of inoculum mixed with wild-type and nolR mutant strains at a concentration of $5 \times 10^{6}$ per milliliter of each strain (ratio $1: 1$ ), and grown under the same condition as described above. After 28 days of cultivation, nodules were randomly picked from each plant root and surface sterilized by immersion in $1 \%$ sodium hypochlorite for $5 \mathrm{~min}$. Nodules were washed three times with sterile water and transferred into a 96-well microtiter plate containing $100 \mu \mathrm{l}$ of TY liquid medium in each well. The surface-sterilized nodule was homogenized by using a sterile toothpick and the suspension was streaked onto TY plates. Three single colonies from each TY plate were transferred onto a TY plates containing $\mathrm{Nm}$ at $150 \mu \mathrm{g} / \mathrm{ml}$. Strains in nodules was determined by $\mathrm{Nm}$ resistance (the wild type was $\mathrm{Nm}$ sensitive and the nolR mutant was Nm resistant).

Quantitative evaluation of competitive nodulation was determined using seven inoculant mixtures, where the proportions of wild-type and nolR mutant strains varied from 1:1,000 to 1,000:1. The mathematical model reported by Amarger and Lobreau (1982) was used to quantify the nodulation competition capability of $S$. medicae nolR mutant strains on $M$. truncatula A17. In this model, a linear relationship exists between the logarithmic ratio of the numbers of nodules formed by each of two strains $\left(N_{\mathrm{A}} / N_{\mathrm{B}}\right)$ and the logarithm of the ratio of the number of cells of the two strains in the inoculum $\left(I_{\mathrm{A}} / I_{\mathrm{B}}\right)$ : $\log \left(N_{\mathrm{A}} / N_{\mathrm{B}}\right) \log C_{\mathrm{AB}}+\mathrm{k} \log \left(I_{\mathrm{A}} / I_{\mathrm{B}}\right)$. The intercept of the regression line, the $C_{\mathrm{AB}}$ value, was reported to be an index representing the competitiveness of strain $\mathrm{A}$ in relation to that of strain B. Moreover, this was considered to be equal to the ratio of the number of nodules formed by strain $A$ to the number of nodules formed by strain $\mathrm{B}$, when the two strains were present in the inoculum in equal quantities. Thus, $C_{\mathrm{AB}}$ should be close to 1 for two strains of equal competitiveness.
Evaluation of nodulation speed by Sinorhizobium strains.

The speed of nodulation and the location of nodules on the primary root (distance from root tip mark) were determined as follows. Briefly, surface-sterilized Medicago seedlings (1- to $2-\mathrm{cm}$ root length) were placed on a paper surface (the paper support from seed growth pouches; Mega International, Minneapolis, MN, U.S.A.) and this was placed on the surface of $1.5 \%$ agar plates (8 to 10 seedlings per plate) containing nitrogen-free Fahräeus medium (Vincent 1970) in square petri dishes (245 by $245 \mathrm{~mm}$; Corning, Corning, NY, U.S.A.). Sinorhizobium inoculants were prepared as described above and $0.1 \mathrm{ml}$ of cell suspensions, adjusted as required by cell densities, were inoculated onto each Medicago seedling root. At the time of inoculation, the location of the root tip was marked on the outside of the petri dish. The dishes were sealed by using surgical tape and the roots were covered with aluminum foil. Petri dishes were placed on a slope in a growth chamber at $25^{\circ} \mathrm{C}$ with a 16 -h light condition and at $21^{\circ} \mathrm{C}$ for $8 \mathrm{~h}$ in the dark. Roots were examined daily for the appearance of nodules and the distances of uppermost nodule from the root tip were recorded at $10 \mathrm{DAI}$. Three replicate petri dishes were prepared for each treatment and the data values were averaged from 20 to 25 plants inoculated with each strain or at each population density.

\section{ACKNOWLEDGMENTS}

This study supported, in part, by grant 0820005 from The National Science Foundation. We thank P. van Berkum and W. Reeve for providing $S$. medicae WSM419 and M. Hynes for providing modified plasmid pFUS1.

\section{LITERATURE CITED}

Amarger, N., and Lobreau, J. P. 1982. Quantitative study of nodulation competitiveness in Rhizobium strains. Appl. Environ. Microbiol. 44:583-588.

Bergersen, F. J. 1982. Root Nodules of Legumes: Structure and Functions. Research Studies Press, Chichester, NY, U.S.A.

Beringer, J. E. 1974. R factor transfer in Rhizobium leguminosarum. J. Gen. Microbiol. 84:188-198.

Bhuvaneswari, T. V., Turgeon, B. G., and Bauer, W. D. 1980. Early events in the infection of soybean (Glycine max L. Merr.) by Rhizobium japonicum: I. Localization of infectible root cells. Plant Physiol. 66:1027-1031.

Bucciarelli, B., Hanan, J., Palmquist, D., and Vance, C. P. 2006. A standardized method for analysis of Medicago truncatula phenotypic development. Plant Physiol. 142:207-219.

Chen, H., Higgins, J., Kondorosi, E., Kondorosi, A., Djordjevic, M. A., Weinman, J. J., and Rolfe, B. G. 2000. Identification of nolR-regulated proteins in Sinorhizobium meliloti using proteome analysis. Electrophoresis 21:3823-3832.

Chen, H., Gao, K., Kondorosi, E., Kondorosi, A., and Rolfe, B. G. 2005. Functional genomic analysis of global regulator NolR in Sinorhizobium meliloti. Mol. Plant-Microbe Interact. 18:1340-1352.

Cren, M., Kondorosi, A., and Kondorosi, E. 1994. An insertional point mutation inactivates NolR repressor in Rhizobium meliloti 1021. J. Bacteriol. 176:518-519.

Cren, M., Kondorosi, A., and Kondorosi, E. 1995. NolR controls expression of the Rhizobium meliloti nodulation genes involved in the core Nod factor synthesis. Mol. Microbiol. 15:733-747.

Crook, M. B., Lindsay, D. P., Biggs, M. B., Bentley, J. S., Price, J. C., Clement, S. C., Clement, M. J., Long, S. R., and Griffitts, J. S. 2012 Rhizobial plasmids that cause impaired symbiotic nitrogen fixation and enhanced host invasion. Mol. Plant-Microbe Interact. 25:1026-1033.

Gordon, D. M., Ryder, M. H., Heinrich, K., and Murphy, P. J. 1996. An experimental test of the rhizopine concept in Rhizobium meliloti. Appl. Environ. Microbiol. 62:3991-3996.

Jitacksorn, S., and Sadowsky, M. J. 2008. Nodulation gene regulation and quorum sensing control density-dependent suppression and restriction of nodulation in the Bradyrhizobium japonicum-soybean symbiosis. Appl. Environ. Microbiol. 74:3749-3756.

Kondorosi, E., Gyuris, J., Schmidt, J., John, M., Duda, E., Hoffmann, B., Schell, J., and Kondorosi, A. 1989. Positive and negative control of nod gene expression in Rhizobium meliloti is required for optimal nodulation. EMBO (Eur. Mol. Biol. Organ.) J. 8:1331-1340. 
Kondorosi, E., Pierre, M., Cren, M., Haumann, U., Buire, M., Hoffmann, B., Schell, J., and Kondorosi, A. 1991. Identification of NolR, a negative transacting factor controlling the nod regulon in Rhizobium meliloti. J. Mol. Biol. 222:885-896.

Liu, R., Tran, V. M., and Schmidt, E. L. 1989. Nodulating competitiveness of a nonmotile Tn7 mutant of Bradyrhizobium japonicum in nonsterile soil. Appl. Environ. Microbiol. 55:1895-1900.

Loh, J., and Stacey, G. 2003. Nodulation gene regulation in Bradyrhizobium japonicum: A unique integration of global regulatory circuits. Appl. Environ. Microbiol. 69:10-17.

Loh, J., Carlson, R. W., York, W. S., and Stacey, G. 2002. Bradyoxetin, a unique chemical signal involved in symbiotic gene regulation. Proc. Natl. Acad. Sci. U.S.A. 99:14446-14451.

Long, S. R. 1989. Rhizobium-legume nodulation: Life together in the underground. Cell 56:203-214.

Long, S. R. 1996. Rhizobium symbiosis: Nod factors in perspective. Plant Cell 8:1885-1898.

Long, S. R., Egelhoff, T., Fisher, R. F., Jacobs, T. W., and Mulligan, J. T. 1985 . Fine structure studies of $R$. meliloti nodDABC genes. Pages 87-94 in: Nitrogen Fixation Research Progress. H. J. Evans, P. J. Bottomley, and W. E. Newton, eds. Martinus Nijhoff Publishers, Boston.

McDermoti, T. R., and Graham, P. H. 1990. Competitive ability and efficiency in nodule formation of strains of Bradyrhizobium japonicum. Appl. Environ. Microbiol. 56:3035-3039.

Miller, J. H. 1972. Experiments in Molecular Genetics. Cold Spring Harbor Laboratory Press, Cold Spring Harbor, NY, U.S.A.

Mulligan, J. T., and Long, S. R. 1985. Induction of Rhizobium meliloti nodC expression by plant exudate requires nodD. Proc. Natl. Acad. Sci. U.S.A. 82:6609-6613

Pongsilp, N., Triplett, E. W., and Sadowsky, M. J. 2005. Detection of homoserine lactone-like quorum sensing molecules in Bradyrhizobium strains. Curr. Microbiol. 51:250-254.

Reeve, W. G., Tiwari, R. P., Wong, C. M., Dilworth, M. J., and Glenn, A. R. 1998. The transcriptional regulator gene phrR in Sinorhizobium meliloti WSM419 is regulated by low $\mathrm{pH}$ and other stresses. Microbiology 144:3335-3342.

Sadowsky, M. J., Bohlool, B. B., and Keyser, H. H. 1987. Serological relatedness of Rhizobium fredii to other rhizobia and to the bradyrhizobia. Appl. Environ. Microbiol. 53:1785-1789.

Sadowsky, M. J., Cregan, P. B., Gottfert, M., Sharma, A., Gerhold, D., Rodriguez-Quinones, F., Keyser, H. H., Hennecke, H., and Stacey, G. 1991. The Bradyrhizobium japonicum nolA gene and its involvement in the genotype-specific nodulation of soybeans. Proc. Natl. Acad. Sci. U.S.A. 88:637-641.

Sambrook, J., and Russell, D. W. 2001. Molecular Cloning: A Laboratory
Manual, 3rd ed. Cold Spring Harbor Laboratory Press, Cold Spring Harbor, NY, U.S.A

Sanchez-Contreras, M., Bauer, W. D., Gao, M., Robinson, J. B., and Allan Downie, J. 2007. Quorum-sensing regulation in rhizobia and its role in symbiotic interactions with legumes. Philos. Trans. R. Soc. Lond. B Biol. Sci. 362:1149-1163.

Simon, R., Priefer, U., and Puhler, A. 1983. A broad host range mobilization system for in vivo genetic engineering: Transposon mutagenesis in gram-negative bacteria. Nat. Biotechnol. 1:784-791.

Streeter, J. G. 1994. Failure of inoculant rhizobia to overcome the dominance of indigenous strains for nodule formation. Can. J. Microbiol. 40:513-522.

Sugawara, M., Epstein, B., Badgley, B. D., Unno, T., Xu, L., Reese, J. Gyaneshwar, P., Denny, R., Mudge, J., Bharti, A. K., Farmer, A. D. May, G. D., Woodward, J. E., Medigue, C., Vallenet, D., Lajus, A., Rouy, Z., Martinez-Vaz, B., Tiffin, P., Young, N. D., and Sadowsky, M. J. 2013. Comparative genomics of the core and accessory genomes of 48 Sinorhizobium strains comprising five genospecies. Genome Biol. 14:R17.

Toro, N. 1996. Nodulation competitiveness in the Rhizobium legume symbiosis. World J. Microbiol. Biotechnol. 12:157-162.

Triplett, E. W., and Sadowsky, M. J. 1992. Genetics of competition for nodulation of legumes. Annu. Rev. Microbiol. 46:399-428.

Uchiumi, T., Ohwada, T., Itakura, M., Mitsui, H., Nukui, N., Dawadi, P., Kaneko, T., Tabata, S., Yokoyama, T., Tejima, K., Saeki, K., Omori, H., Hayashi, M., Maekawa, T., Sriprang, R., Murooka, Y., Tajima, S., Simomura, K., Nomura, M., Suzuki, A., Shimoda, Y., Sioya, K., Abe, M., and Minamisawa, K. 2004. Expression islands clustered on the symbiosis island of the Mesorhizobium loti genome. J. Bacteriol. 186:2439-2448.

Vieira, J., and Messing, J. 1982. The pUC plasmids, an M13mp7-derived system for insertion mutagenesis and sequencing with synthetic universal primers. Gene 19:259-268.

Vinardell, J. M., Ollero, F. J., Hidalgo, A., Lopez-Baena, F. J., Medina, C., Ivanov-Vangelov, K., Parada, M., Madinabeitia, N., Espuny Mdel, R. Bellogin, R. A., Camacho, M., Rodriguez-Navarro, D. N., Soria-Diaz, M. E., Gil-Serrano, A. M., and Ruiz-Sainz, J. E. 2004. NolR regulates diverse symbiotic signals of Sinorhizobium fredii HH103. Mol. PlantMicrobe Interact. 17:676-685.

Vincent, J. M. 1970. A Manual for the Practical Study of Root-Nodule Bacteria, IBP Handb. No. 15, Blackwell Scientific Press, Oxford.

Yuhashi, K., Ichikawa, N., Ezura, H., Akao, S., Minakawa, Y., Nukui, N., Yasuta, T., and Minamisawa, K. 2000. Rhizobitoxine production by Bradyrhizobium elkanii enhances nodulation and competitiveness on Macroptilium atropurpureum. Appl. Environ. Microbiol. 66:2658-2663. 